\title{
A Suboptimal Estimator Design for Nonlinear Systems Using Covariance Matching
}

\author{
Jang Gyu LEE* and Yeon Seok LEE**
}

A new finite dimensional approximation filter is derived for nonlinear systems. For the finite dimensional approximation, a statistical linearization method is employed. The proposed filter is compared with other statistical linearization filter and the extended Kalman filter using an example.

Key Words : nonlinear estimator, stochastic linearization

\section{Introduction}

In linear systems with Gaussion process, Kalman filter is known to be extremely useful as an optimal estimator in a wide variety of practical applications ${ }^{1,2)}$. However, most physical systems are modelled as nonlinear systems. Optimal estimation problem for nonlinear systems is very complicated and usually infinite dimensional ${ }^{3}$. Therefore, several methods of finite approximations to preserve some of the important properties of the optimal estimator, are inevitable. The extended Kalman filter is constructed by the truncated Taylor series expansions and is widely accepted and applied to a large class of nonlinear estimation problems ${ }^{2}$. Phaneuf ${ }^{4)}$ and Sunahara ${ }^{5}$ derived linearized filter using statistical linearization technique of random input describing functions (RIDF's) ${ }^{6) ~ 7)}$. These methods assume that all processes have Gaussian distributions. In this note, a suboptimal estimator under Gaussian assumption is derived and described by a new statistical linearization technique. This technique is based on an exact mean and variance information of nonlinear function:

A new statistical linearization technique is introduced in section 2. The derivation of the proposed estimator using this technique is described in section 3 , and some simulated results compared to the extended Kalman filter and the RIDF's estimator are given in section 4. Discussions of the simulation results are described in section 5 .

\section{Statistical Linearization}

Consider a linear approximation for a vector function $\boldsymbol{f}(\boldsymbol{x})$ in Eq. (1) by a linear expression in Eq. (2).

\footnotetext{
* Seoul National University, San 56-1, Shinrim-Dong Kwanak-ku, Seoul, 151-742, KOREA

** Kunsan National University, San 68, Miryong-Dong, Kunsan, Chonbuk, 573-360, KOREA

(Received June 22, 1993)
}

$$
\begin{aligned}
& \boldsymbol{y}=\boldsymbol{f}(\boldsymbol{x}) \\
& \tilde{\boldsymbol{y}}=\boldsymbol{M}+C(\boldsymbol{x}-\boldsymbol{m})
\end{aligned}
$$

This representation means that the distribution of $\boldsymbol{y}$ is also Gaussian. A new statistical linearization technique, the covariance matching method, is to match the mean and covariance of $\boldsymbol{y}$ with the mean and covariance of $\tilde{\boldsymbol{y}}$. The output mean $\boldsymbol{M}$ and random gain $C$ should satisfy

$$
\begin{aligned}
& E\{\boldsymbol{f}(\boldsymbol{x})\}=E\{\boldsymbol{M}+C(\boldsymbol{x}-\boldsymbol{m})\}=\boldsymbol{M} \\
& E\left\{(\boldsymbol{y}-E\{\boldsymbol{y}\})(\boldsymbol{y}-E\{\boldsymbol{y}\})^{T}\right\} \\
& \quad=E\left\{(\tilde{\boldsymbol{y}}-E\{\tilde{\boldsymbol{y}}\})(\tilde{\boldsymbol{y}}-E\{\tilde{\boldsymbol{y}}\})^{T}\right\} \\
& \quad=E\left\{(\boldsymbol{M}+C(\boldsymbol{x}-\boldsymbol{m})-\boldsymbol{M})(\boldsymbol{M}+C(\boldsymbol{x}-\boldsymbol{m})-\boldsymbol{M})^{T}\right\} \\
& \boldsymbol{M}=E\{\tilde{\boldsymbol{y}}\}=E\{\boldsymbol{y}\} \\
& C E\left\{(\boldsymbol{x}-\boldsymbol{m})(\boldsymbol{x}-\boldsymbol{m})^{T}\right\} C^{T} \\
& \quad=E\left\{(\boldsymbol{y}-E\{\boldsymbol{y}\})(\boldsymbol{y}-E\{\boldsymbol{y}\})^{T}\right\} .
\end{aligned}
$$

This approximation is different from functional linearization. The random component of $\boldsymbol{y}$ is denoted by that of input $x$ multiplied by random gain $C$. There may be many methods to obtain the $c_{i j}$ with a proper meaning.

\section{Conditional Minimum Variance Estimator}

Consider a nonlinear system in Eq. (5) with a discrete measurement $\boldsymbol{y}_{k}$.

$$
\begin{aligned}
& \dot{\boldsymbol{x}}_{t}=\boldsymbol{f}\left(\boldsymbol{x}_{t}, t\right)+G_{t} \boldsymbol{w}_{t}, \boldsymbol{x}_{t_{0}} \sim \mathcal{N}\left(\boldsymbol{m}_{0}, P_{0}\right), \\
& \boldsymbol{w}_{t} \sim \mathcal{N}\left(\boldsymbol{b}_{t}, Q_{t}\right), \\
& \boldsymbol{y}_{k}=\boldsymbol{h}\left(\boldsymbol{x}_{k}, t_{k}\right)+\boldsymbol{v}_{k}, \boldsymbol{v}_{\boldsymbol{k}} \sim \mathcal{N}\left(\mathbf{0}, R_{k}\right),
\end{aligned}
$$

For simplicity, $\left\{\boldsymbol{w}_{t}\right\}$ and $\left\{\boldsymbol{v}_{k}\right\}$ are assumed independent, and $\left\{\boldsymbol{w}_{t}\right\}$ is independent of $\boldsymbol{x}_{0}$. The objective is to obtain a finite dimensional approximations of the mean and covariance conditioned on the set of discrete measurements $Y_{n}=\left\{\boldsymbol{y}_{1}, \boldsymbol{y}_{2}, \cdots, \boldsymbol{y}_{n}\right\}$ under Gaussian assumption.

Consider a minimum variance estimate $\widehat{x}_{k-1}$ with its covariance $P_{k-1}$ conditioned on $Y_{k-1}$. Because the probability density function of Gaussian distribution is unimodal and symmetric about its mean, the mean $\bar{x}_{t}$ conditioned on $Y_{k-1}$ is the minimum variance estimate for the time interval $t_{k-1} \leq t<t_{k}$. The conditional mean and covariance are propagated through the system dynamics to the next measurements $\boldsymbol{y}_{k}$ at 
time $t_{k}$.

$$
\begin{aligned}
\dot{\bar{x}}_{t}= & E\left\{\dot{\boldsymbol{x}}_{t} \mid Y_{k-1}\right\}=E\left\{\boldsymbol{f}\left(\boldsymbol{x}_{t}, t\right)+G_{t} \boldsymbol{w}_{t} \mid Y_{k-1}\right\} \\
= & =E\left\{\boldsymbol{f}\left(\boldsymbol{x}_{t}, t\right) \mid Y_{k-1}\right\}+G_{t} \boldsymbol{b}_{t} \\
\dot{\dot{P}_{t}}= & \frac{\partial}{\partial t} E\left\{\left(\boldsymbol{x}_{t}-\overline{\boldsymbol{x}}_{t}\right)\left(\boldsymbol{x}_{t}-\overline{\boldsymbol{x}}_{t}\right)^{T} \mid Y_{k-1}\right\} \\
= & E\left\{\left(\boldsymbol{f}_{t}-\dot{\bar{x}}_{t}\right)\left(\boldsymbol{x}_{t}-\overline{\boldsymbol{x}}_{t}\right)^{T} \mid Y_{k-1}\right\} \\
& +E\left\{\left(\boldsymbol{x}_{t}-\overline{\boldsymbol{x}}_{t}\right)\left(\boldsymbol{f}_{t}-\overline{\bar{x}}_{t}\right)^{T} \mid Y_{k-1}\right\}+G_{t} Q_{t} G_{t}^{T}
\end{aligned}
$$

Assume that the update structure be a linear form of the new information obtained from new measurements as shown in Eq. (10). Then, the remaining problem is to choose the estimate gain $K$ to minimize a scalar sum of the diagonal elements of updated covariance matrix of Eq. (9).

$$
\begin{aligned}
J_{k} & =E\left\{\left(\boldsymbol{x}_{k}-\widehat{\boldsymbol{x}}_{k}\right)^{T}\left(\boldsymbol{x}_{k}-\widehat{\boldsymbol{x}}_{k}\right) \mid Y_{k}\right\} \\
\tilde{\boldsymbol{x}}_{k} & =E\left\{\boldsymbol{x}_{k} \mid Y_{k}\right\} \\
& =\overline{\boldsymbol{x}}_{k}+K \widetilde{\boldsymbol{y}}_{k} \\
\tilde{\boldsymbol{y}}_{k} & =\boldsymbol{y}_{k}-E\left\{\boldsymbol{h}\left(\boldsymbol{x}_{k}\right) \mid Y_{k-1}\right\}
\end{aligned}
$$

Substitution of $\widehat{x}_{k}$ in Eq. (10) into Eq. (9) generates $J_{k}$ as the function of $K$, i. e.,

$$
\begin{aligned}
J_{k}= & E\left\{\left(\boldsymbol{x}_{k}-\overline{\boldsymbol{x}}_{k}\right)^{T}\left(\boldsymbol{x}_{k}-\overline{\boldsymbol{x}}_{k}\right) \mid Y_{k}\right\} \\
& -2 E\left\{\left(\boldsymbol{x}_{k}-\overline{\boldsymbol{x}}_{k}\right)^{T} K \tilde{\boldsymbol{y}}_{k} \mid Y_{k}\right\} \\
& +E\left\{\left(K \tilde{\boldsymbol{y}}_{k}\right)^{T}\left(K \tilde{\boldsymbol{y}}_{k}\right) \mid Y_{k}\right\} .
\end{aligned}
$$

Because the Hessian of $J_{k}$ is always positive definite, $J_{k}$ has the global minimum value. The gain $K$ minimizing the performance index $J_{k}$ is obtained by

$$
K=E\left\{\left(\boldsymbol{x}_{k}-\overline{\boldsymbol{x}}_{k}\right) \tilde{\boldsymbol{y}}_{k}^{T} \mid Y_{k}\right\} E\left\{\tilde{\boldsymbol{y}}_{k} \tilde{\boldsymbol{y}}_{k}^{T} \mid Y_{k}\right\}^{-1}
$$

and the minimum variance is given by Eq. (14) after some manipulation.

$$
P_{k}=\bar{P}_{k}-K E\left\{\tilde{\boldsymbol{y}}_{k}\left(\boldsymbol{x}_{k}-\overline{\boldsymbol{x}}_{k}\right)^{T} \mid Y_{k}\right\}
$$

Therefore, the propagation of Eqs. (7) and (8) and the update of Eqs. (11), (13), (10) and (14) construct an optimal estimator. But, this implementation requires some approximations. Direct calculation of these expectations under Gaussian assumption produces a finite dimensional algorithm.

The expectation of $E\left\{\boldsymbol{f}\left(\boldsymbol{x}_{t}, t\right) \mid Y_{k-1}\right\}$ in Eq. ( 7$)$ is propagated from $x_{k-1}$ under the assumption of normal distribution with its mean $\bar{x}_{k-1}$ and its covariance $P_{k-1}$, and still $\overline{\boldsymbol{x}}_{t}$ propagated from $\overline{\boldsymbol{x}}_{k-1}$ preserves normally distributed assumption. Consequently, the expectations conditioned on the measurements set $Y_{k-1}$ or $Y_{k}$ can be denoted in the same way.

$$
\begin{aligned}
E\left\{\boldsymbol{f}\left(\boldsymbol{x}_{t}, t\right) \mid Y_{\boldsymbol{k}-1}\right\} & =E\left\{\boldsymbol{f}\left(\boldsymbol{x}_{t}, t\right) \mid x_{t} \sim \mathcal{N}\left(\overline{\boldsymbol{x}}_{t}, \bar{P}_{t}\right)\right\} \\
& =\boldsymbol{M}_{\boldsymbol{f}}\left(\overline{\boldsymbol{x}}_{t}, \bar{P}_{t}\right)
\end{aligned}
$$

And the same procedure makes the mean of measurement $\widetilde{\boldsymbol{y}}_{\boldsymbol{k}}$ be $\boldsymbol{M}_{\boldsymbol{h}}$ in Eq. (16). The conditional correlation between $\boldsymbol{x}_{t}$ and $\boldsymbol{f}_{t}$ in Eq. ( 8 ) is represented by a random gain of RIDF like Eq. (17), and the correlation between $\boldsymbol{x}_{t}$ and $\boldsymbol{h}_{t}$ in Eq. (14) like Eq. (18). Finally the covariance of the measurement in the estimator gain $K$ is represented by a random gain of the covariance matching technique like Eq. (19).

$$
\begin{aligned}
& E\left\{\boldsymbol{h}\left(\boldsymbol{x}_{t}, t\right) \mid Y_{k-1}\right\}=\boldsymbol{M}_{\boldsymbol{h}}\left(\overline{\boldsymbol{x}}_{t}, \bar{P}_{t}\right) \\
& E\left\{\left(\boldsymbol{f}_{t}-E\left\{\boldsymbol{f}_{t} \mid Y_{k-1}\right\}\right)\left(\boldsymbol{x}_{t}-E\left\{\boldsymbol{x}_{t} \mid Y_{k-1}\right\}\right)^{T} \mid Y_{k-1}\right\} \\
& \quad=N_{\boldsymbol{f}}\left(\overline{\boldsymbol{x}}_{t}, \bar{P}_{t}\right) \bar{P}_{t}
\end{aligned}
$$

$$
\begin{aligned}
& E\left\{\left(\boldsymbol{h}_{t}-E\left\{\boldsymbol{h}_{t} \mid Y_{k-1}\right\}\right)\left(\boldsymbol{x}_{t}-E\left\{\boldsymbol{x}_{t} \mid Y_{k-1}\right\}\right)^{T} \mid Y_{k-1}\right\} \\
& \quad=N_{\boldsymbol{h}}\left(\overline{\boldsymbol{x}}_{t}, \bar{P}_{t}\right) \bar{P}_{t} \\
& E\left\{\left(\boldsymbol{h}_{t}-\boldsymbol{M}_{\boldsymbol{h}}\left(\overline{\boldsymbol{x}}_{t}, \bar{P}_{t}\right)\right)\left(\boldsymbol{h}_{t}-\boldsymbol{M}_{\boldsymbol{h}}\left(\overline{\boldsymbol{x}}_{t}, \bar{P}_{t}\right)\right)^{T} \mid Y_{\boldsymbol{k}-1}\right\} \\
& \quad=C_{\boldsymbol{h}}\left(\overline{\boldsymbol{x}}_{k}, \bar{P}_{\boldsymbol{k}}\right) \bar{P}_{\boldsymbol{k}} C_{\boldsymbol{h}}\left(\overline{\boldsymbol{x}}_{k}, \bar{P}_{\boldsymbol{k}}\right)^{T}
\end{aligned}
$$

Especially, these expectations can be described by the mean and covariance because of Gaussian assumption. That is why the proposed filter in this note is suboptimal, since there is no guarantee that a nonlinear system can hold Gaussian assumptions.

\section{Simulations}

Consider a nonlinear dynamical system of Eq. (20) with its measurement sequences Eq. (21).

$$
\begin{aligned}
& \dot{x}_{t}=-x_{t}-a x_{t}^{3}+\bar{w}_{t} \\
& y_{k}=\sin x_{t_{k}}+v_{k} \\
& x_{0} \sim \mathcal{N}\left(m_{0}, P_{0}\right), w_{t} \sim \mathcal{N}\left(0, Q_{t}\right), v_{k} \sim \mathcal{N}\left(0, R_{k}\right), \\
& E\left\{w_{t} v_{j}\right\}=0
\end{aligned}
$$

Bearing in mind the Gaussian assumption in every step, application of the proposed estimate algorithm from (7) to (14) to the present example gives the filter equations as follows.

$$
\begin{aligned}
& \dot{\bar{x}}_{t}=-\bar{x}_{t}-a \bar{x}_{t}^{3}-3 a \bar{x}_{t} \bar{P}_{t} \\
& \dot{\bar{P}}_{t}=\left(-2-6 a \bar{x}_{t}^{2}-3 a \bar{P}_{t}\right) \bar{P}_{t}+Q_{t} \\
& \tilde{y}_{k}=y_{k}-e^{\left(-\bar{P}_{k} / 2\right)} \sin \left(\bar{x}_{t}\right) \\
& K=\frac{\bar{P}_{k} e^{-\left(\bar{P}_{k} / 2\right)} \cos \left(\bar{x}_{t}\right)}{0.5-e^{\left(-2 \bar{P}_{k}\right)} \cos \left(2 \bar{x}_{t}\right)-e^{\left(-\bar{P}_{k}\right)} \sin ^{2}\left(\bar{x}_{t}\right)+R_{k}} \\
& \hat{x}=\bar{x}_{k}+K \tilde{y}_{k} \\
& P_{k}=\bar{P}_{k}-K e^{-\left(\bar{P}_{k} / 2\right)} \cos \left(\bar{x}_{t}\right) \bar{P}_{k}
\end{aligned}
$$

A variety of single and averaged runs have been made, and compared with the RIDF estimator designed by Phaneuf and the extended Kalman filter. In simulations all nonlinear integrations are carried out by the Runge-Kutta 4 -th order method with constant step-size 0.01 second. In Fig. 1 the measurement and system noise covariances are given by 0.2 and $a$ $=0.4$. The true state starts from 0.7 while the filter initial value is given by 1.5 with covariance 0.3 . Simulation result shows that all estimates trace the true state well in steady state. But each represents different transient actions with noticeable overshoots. The extended Kalman filter and the RIDF estimator have a large overshoot. In the proposed estimator, however, the overshoot is not seen at all. Fig. 2, another single run result with different random seed number, shows the divergence of the extended $\mathrm{Kal}$ man filter. The RIDF estimator has a larger overshoot than the previous run. Another result in Fig. 3 shows divergence of two estimators, while the proposed estimator maintains the same curve. An interesting fact is that the RIDF estimator rebalances a large initial overshoot.

The above simulation results show another problems of nonlinear system. Too a large gain in starting point makes the extended Kalman filter and the RIDF estimator diverge. Especially, the condition of 
CONVERGENCE WITH LARGE OVERSHOOT

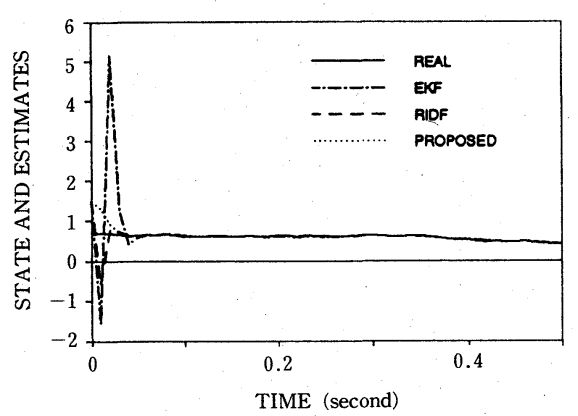

Fig. 1 Convergence Property I

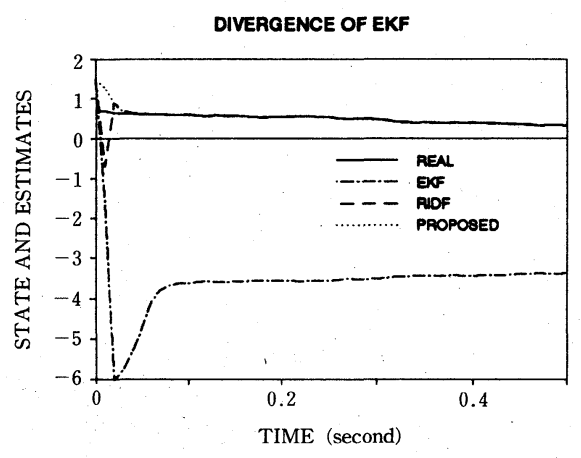

Fig. 2 Convergence Property II

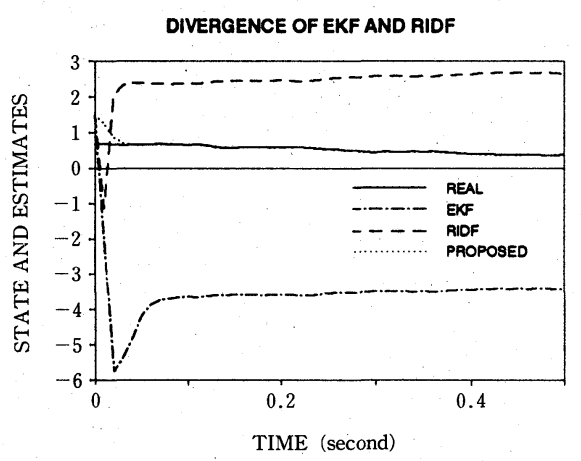

Fig. 3 Convergence Property III

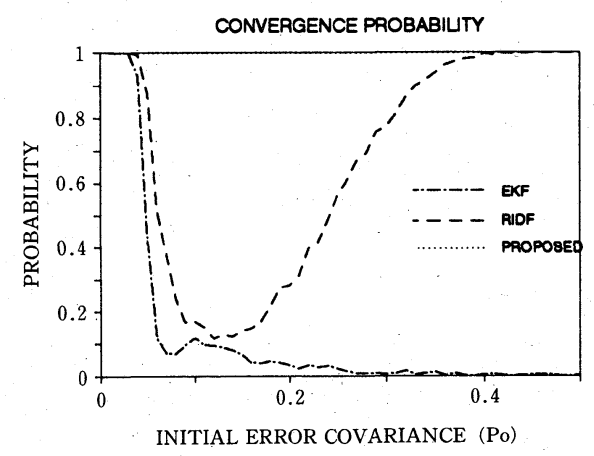

Fig. 4 Effect of initial error covariance the simulations represents small correlation between the state and its measurement. The small correlation results in small covariance of its residual, and eventually a large gain. On the contrary, the proposed estimator, since the gain is computed from an exact residual covariance, is less sensitive to the operating condition. That is why the proposed method exhibits robust to change in initial conditions. In order to demonstrate the superiority of the proposed estimator over the other estimators, the convergence probabilities from 300 simulation runs are calculated and depicted in Fig. 4. The initial error covariance has been varied from 0.001 to 0.5 . Other conditions are same as those in Fig. 1. The RIDF estimator has larger convergent probability under the same initial error covariance than the extended Kalman filter, and its probability grows as initial error covariance grows. The proposed estimator has always probability 1 under the given conditions.

\section{Conclusion}

The basic idea of the approximation filter developed here is the exact calculation of all expectation operators of the conditional probability density function under Gaussian assumption. The Gaussian assumption implies that the infinite dimensional estimator is approximated by the two dimensional estimator consisting of the first and second order moments. Although it is difficult to justify analytically the accuracy of stochastic linearization methods described here, numerical results reveal that the proposed method will play a useful role for nonlinear estimation problems.

\section{References}

1) A. Gelb: Applied Optimal Estimation, Cambridge, Mass, MIT Press (1974)

2) A. H. Jazwinski : Stochastic Processes and Filtering Theory, New York, Academic (1970)

3) H. J. Kushner: Approximations of Optimal Nonlinear Filters, IEEE Trans., AC-12-8, 546/556 (1967)

4) Roger J. Phaneuf : Approximate nonlinear estimation, Ph. D. Thesis, Massachusetts Institute of Technology, May 1 (1968)

5) Y. Sunahara: An approximate method of state estimation for nonlinear dynamical systems, Trans. ASME, J. Basic Eng., 92-1, 385/393 (1970)

6) Richard C. Booton, Jr. : Nonlinear Control Systems with Random Inputs, Transactions of the IRE, Professional Group on Circuit Theory, CT-1-1, March 2/7 (1954)

7) Gelb, A. and R. S. Warren: Direct statistical analysis of nonlinear systems: CADET, AIAA Journal, 11-5, 689/694 (1973) 